\title{
Combined measurement of carbon monoxide and nitric oxide lung transfer does not improve the identification of pulmonary hypertension in systemic sclerosis
}

\author{
Bruno Degano ${ }^{1,2}$, Thibaud Soumagne ${ }^{2}$, Thomas Delaye ${ }^{3}$, Patrick Berger ${ }^{4}$, \\ Thierry Perez ${ }^{5}$, Alicia Guillien ${ }^{1,2}$, Jean-Luc Pellegrin ${ }^{6}$, David Launay ${ }^{7,8}$, \\ Nadine Magy-Bertrand ${ }^{9}$, Christian Agard ${ }^{10}$, Kiet Phong Tiev ${ }^{11}$, Thông Hua-Huy ${ }^{12}$, \\ Catherine Tardiff ${ }^{13}$, Véronique Diaz ${ }^{14}$, Arnaud Chambellan ${ }^{15,16}$ and \\ Anh Tuan Dinh-Xuan ${ }^{12,16}$
}

@ERSpublications

Compared with TLCO alone, combined TLCO-TLNO measurement does not improve detection of PH in unselected SSc patients http://ow.ly/ITHO30eldMk

Cite this article as: Degano B, Soumagne T, Delaye T, et al. Combined measurement of carbon monoxide and nitric oxide lung transfer does not improve the identification of pulmonary hypertension in systemic sclerosis. Eur Respir J 2017; 50: 1701008 [https://doi.org/10.1183/13993003.01008-2017].

ABSTRACT Screening is important to determine whether patients with systemic sclerosis (SSc) have pulmonary hypertension because earlier pulmonary hypertension treatment can improve survival in these patients. Although decreased transfer factor of the lung for carbon monoxide (TLCO) is currently considered the best pulmonary function test for screening for pulmonary hypertension in SSc, small series have suggested that partitioning TLCO into membrane conductance (diffusing capacity) for carbon monoxide (DMCO) and alveolar capillary blood volume $(V C)$ through combined measurement of TLCO and transfer factor of the lung for nitric oxide (TLNO) is more effective to identify pulmonary hypertension in SSc patients compared with TLCO alone. Here, the objective was to determine whether combined TLCOTLNO partitioned with recently refined equations could more accurately detect pulmonary hypertension than TLCO alone in SSc.

For that purpose, 572 unselected consecutive SSc patients were retrospectively recruited in seven French centres.

Pulmonary hypertension was diagnosed with right heart catheterisation in 58 patients. TLCO, TLNO and VC were all lower in SSc patients with pulmonary hypertension than in SSc patients without pulmonary hypertension. The area under the receiver operating characteristic curve for the presence of pulmonary hypertension was equivalent for TLCO $(0.82,95 \%$ CI $0.79-0.85)$ and TLNO (0.80, 95\% CI $0.76-0.83)$, but lower for $V \mathrm{C}(0.75,95 \%$ CI $0.71-0.78)$ and DMCO (0.66, 95\% CI 0.62-0.70).

Compared with TLCO alone, combined TLCO-TLNO does not add capability to detect pulmonary hypertension in unselected SSc patients.

Received: May 172017 | Accepted after revision: July 202017

Conflict of interest: None declared.

This article has supplementary material available from erj.ersjournals.com

Copyright @ERS 2017 
Affiliations: ${ }^{1} E A$ 3920, Université de Franche-Comté, Besançon, France. ${ }^{2}$ Service d'Explorations Fonctionnelles Respiratoires, CHRU de Besançon, Besançon, France. ${ }^{3}$ Service de Médecine Interne, CHU de Poitiers, Poitiers, France. ${ }^{4}$ Service d'Explorations Fonctionnelles Respiratoires, CHU de Bordeaux, Pessac, France. ${ }^{5}$ Service d'Explorations Fonctionnelles Respiratoires, CHU de Lille, Lille, France. ${ }^{6}$ Service de Médecine Interne, CHU de Bordeaux, Pessac, France. ${ }^{7}$ Dépt de Médecine Interne et Immunologie Clinique, $\mathrm{CHU}$ de Lille, Lille, France. ${ }^{8}$ Lille Inflammation Research International Center (LIRIC), U995, Université de Lille, Lille, France. ${ }^{9}$ Service de Médecine Interne, CHRU de Besançon, Besançon, France. ${ }^{10}$ Service de Médecine Interne, Centre de Compétence Maladies Systémiques et Auto-immunes Rares, CHU de Nantes, Nantes, France. ${ }^{11}$ Service de Médecine Interne, Hôpital Saint Antoine, Université Pierre et Marie Curie, Paris, France. ${ }^{12}$ Service de Physiologie Respiratoire, Hôpital Cochin, Université Paris Descartes, Paris, France. ${ }^{13}$ Service de Physiologie Digestive, Urinaire, Respiratoire et Sportive, $\mathrm{CHU}$ de Rouen, Rouen, France. ${ }^{14}$ Service d'Explorations Fonctionnelles Respiratoires and équipe ALIVE (CIC 1402), CHU de Poitiers, Poitiers, France. ${ }^{15}$ Institut du Thorax, CHU de Nantes, Nantes, France. ${ }^{16}$ These two authors contributed equally to this work.

Correspondence: Bruno Degano, Physiologie-Explorations Fonctionnelles, CHU Jean Minjoz, 25030 Besançon, France. E-mail: bruno.deganoduniv-fcomte.fr

\section{Introduction}

Systemic sclerosis (SSc) is a connective tissue disease characterised by widespread vasculopathy and excessive fibrosis in multiple organs, including the skin, digestive tract, kidneys, heart and lungs [1]. Since the early 1980s, with the advent of angiotensin-converting enzyme inhibitors that have improved the treatment of scleroderma renal crisis, lung diseases have become the leading cause of death in SSc [2]. Interstitial lung disease (ILD) and pulmonary hypertension are the two main forms of pulmonary involvement in SSc [3]. Interstitial abnormalities on high-resolution computed tomography (HRCT) are found in many scleroderma patients, but lesions are generally of limited extent [4], and early deaths attributable to ILD are uncommon in SSc [5, 6]. Although less frequent than ILD, pulmonary hypertension either isolated or associated with ILD has a poor prognosis in SSc [3]. Early diagnosis and prompt therapy of pulmonary hypertension are beneficial from a prognostic standpoint in SSc patients either with or without ILD $[7,8]$, and recommendations for active screening of pulmonary hypertension in SSc have therefore been made [9].

Several pulmonary function tests are widely used for screening of pulmonary hypertension. Among them, impaired transfer factor of the lung for carbon monoxide (TLCO) has been recognised as the most sensitive marker of pulmonary hypertension in SSc $[10,11]$. Nevertheless, this test lacks specificity, at least in part because ILD contributes to altering TLCO [12]. In order to overcome this lack of specificity, a "partition" of TLCO into two transfer components, i.e. membrane conductance (diffusing capacity) for carbon monoxide (DMCO) and alveolar capillary blood volume $(V C)$, has been proposed [13]. A decreased DMCO is interpreted as a "thickening" of the alveolo-capillary membrane or as a decrease in lung area, while a decreased $V C$ is considered to represent reduced blood volume in the ventilated alveoli [14]. Partitioning TLCO can be calculated with the Roughton-Forster equation either by measuring TLCO at several oxygenation levels or by combining measurement of TLCO with transfer factor of the lung for nitric oxide (TLNO) [15-17]. Several studies have evaluated the diagnostic power of DMCO and VC to identify pulmonary hypertension in SSc patients. Yet, to date, only small, single-centre series of SSc patients have been studied and results have been predominantly contradictory [18-20]. In addition, the equations used for the calculation of DMCO and VC with combined TLCO-TLNO in these studies are currently viewed as inappropriate $[16,21]$.

Therefore, in a large, multicentre sample of unselected patients with SSc who had combined TLCO-TLNO measurements, we aimed to assess the usefulness of the TLCO partition into DMCO and VC for the identification of pulmonary hypertension, with $D \mathrm{MCO}$ and $V_{\mathrm{C}}$ being calculated according to the latest equations proposed by the European Respiratory Society (ERS) Task Force [16]. We also aimed to study whether or not calculation of DMCO and $V$ C helps to predict overall survival in patients with SSc.

\section{Methods}

Patients

Consecutive unselected patients with SSc were retrospectively recruited in seven French medical centres (Besançon, Bordeaux, Paris-Cochin, Lille, Nantes, Poitiers and Rouen). Patients were evaluated in stable conditions. The diagnosis of SSc was made or confirmed by a specialist according to current recommendations [22]. Inclusion date was the date of measurement of combined TLCO-TLNO. Clinical information was retrospectively collected for all participating patients, and included cutaneous disease subset (according to the latest recommendations [22]), disease duration (from the date of first disease manifestation other than Raynaud's phenomenon) and history of digital ulceration. Biochemical and serological blood tests, including haemoglobin, antinuclear antibodies, anticentromere antibodies and antitopoisomerase I antibodies, were also collected. ILD was assessed by the presence of subpleural 
ground-glass opacities and/or interstitial reticular pattern with or without traction bronchiectasis, and/or honeycomb cysts on HRCT $[4,20]$.

The study was approved by the Institutional Review Board of the French Language Society for Respiratory Medicine (Société de Pneumologie de Langue Française), which deemed it to be observational and therefore waived the need for informed consent (CEPRO 2015-025).

\section{Assessment of pulmonary hypertension}

All patients underwent Doppler echocardiography at the time of pulmonary function tests, for the measurement of left ventricular ejection fraction and tricuspid regurgitant jet velocity. Right heart catheterisation was performed in patients with tricuspid regurgitant jet velocity $>3 \mathrm{~m} \cdot \mathrm{s}^{-1}$ and in patients with unexplained dyspnoea along with tricuspid regurgitant jet velocity $>2.5 \mathrm{~m} \cdot \mathrm{s}^{-1}$ [11]. A diagnosis of pulmonary hypertension was retained in patients with a mean pulmonary artery pressure $\geqslant 25 \mathrm{mmHg}$ together with a mean pulmonary arterial wedge pressure $\leqslant 15 \mathrm{mmHg}$ measured during right heart catheterisation performed at rest [7].

\section{Pulmonary function tests}

Forced expiratory flow in $1 \mathrm{~s}$, forced vital capacity, slow vital capacity, functional residual capacity, residual volume and total lung capacity were assessed with standard pulmonary function test equipment in each participating centre according to the ERS guidelines [23, 24]. References values for spirometry and lung volumes were from QUANJER et al. [25].

Combined TLCO-TLNO measurements were performed with a commercially available device following daily calibration (Medisoft, Dinant, Belgium), according to a procedure described elsewhere [14]. Calculations of $D M C O$ and $V C$ were made according to the most recent recommendations (see supplementary material) [16]. Reference values for TLCO, TLNO, VC and DMCO were from Zavorsky et al. [16].

\section{Statistical analyses}

Patients were first divided into four groups according to the results of ILD and pulmonary hypertension assessments: SSc patients without pulmonary involvement (Group A), SSc patients with ILD alone (Group B), SSc patients with both ILD and pulmonary hypertension (Group C), and SSc patients with pulmonary hypertension alone (Group D). Data are presented as mean \pm SD for quantitative variables and as percentages for categorical variables. Comparisons of categorical data between groups were performed using Fisher's exact test. ANOVA was performed to evaluate differences of quantitative variables between the four groups. Scheffe's tests were applied to evaluate pairwise comparisons when a significant difference was found by ANOVA. The Kaplan-Meier method was used to illustrate survival according to the group, with statistically significant differences in event rates assessed via the log-rank test.

Analyses were then performed between SSc patients without pulmonary hypertension (Groups A and B) and SSc patients with pulmonary hypertension (Groups $\mathrm{C}$ and D). The area under the receiver operating characteristic (ROC) curve (AUC) was used to assess the ability of TLCO, TLNO, VC, DMCO, TLNO/TLCO and $\mathrm{DMCO} / \mathrm{VC}$ to discriminate between the presence and absence of pulmonary hypertension. The optimal threshold for the identification of pulmonary hypertension was assessed according to the Youden index of ROC curve analysis in order to maximise both sensitivity and specificity [26]. Comparisons of AUC values were performed with MedCalc Statistical Software version 16.8.4 (MedCalc, Ostend, Belgium). Univariate analysis based on the proportional hazards model was used to examine the relationship between survival and Tlco, TLNo, DMCO, VC and relevant clinical data. Multivariate analysis using Cox's proportional hazards regression model was used to examine the independent effect of each of these variables on survival. Variables with $\mathrm{p}<0.20$ in the univariate analysis were included in the multivariate model.

A p-value of 0.05 was deemed to be statistically significant. All analyses except comparisons of AUC values were performed with SAS version 9.4 (SAS Institute, Cary, NC, USA).

\section{Results}

\section{Patient characteristics}

We identified a total of $572 \mathrm{SSc}$ patients who had combined TLCO-TLNO measurements between November 2006 and April 2016 in the seven participating centres. The main characteristics of the four groups of patients, divided according to the results of pulmonary hypertension and of ILD assessments, are given in table 1.

\section{Pulmonary function tests}

TLCO and TLNO were the lowest in SSc patients with pulmonary hypertension (Groups C and D), and were also lower in SSc patients with ILD (Group B) than in SSc patients without any pulmonary involvement 
TABLE 1 Main characteristics at inclusion of patients with systemic sclerosis (SSC), divided into four groups: SSc patients without any pulmonary involvement, i.e. neither interstitial lung disease (ILD) nor pulmonary hypertension (Group A), SSc patients with ILD but without pulmonary hypertension (Group B), SSc patients with both ILD and pulmonary hypertension (Group C), and SSc patients without ILD but with pulmonary hypertension (Group D)

\begin{tabular}{|c|c|c|c|c|c|}
\hline & Group A & Group B & Group C & Group D & p-value \\
\hline Subjects & 313 & 201 & 35 & 23 & \\
\hline Age years & $56 \pm 14^{\pi,+}$ & $58 \pm 13^{+}$ & $63 \pm 11$ & $67 \pm 10$ & $<0.0001$ \\
\hline BMI $\mathrm{kg} \cdot \mathrm{m}^{-2}$ & $25 \pm 5$ & $24 \pm 4$ & $26 \pm 5$ & $24 \pm 5$ & 0.33 \\
\hline Female & $268(86)^{\#, 9}$ & $148(74)$ & $21(60)$ & $20(87)$ & 0.0001 \\
\hline Ever-smoker & $113(36)$ & 70 (35) & $10(29)$ & $5(22)$ & 0.72 \\
\hline Cutaneous subtype & & & & & $<0.0001$ \\
\hline Limited & $277(88)^{\#, 9}$ & $106(53)^{+}$ & $17(49)^{+}$ & $21(91)$ & \\
\hline Diffuse & $36(12)^{\#, 9}$ & $95(47)^{+}$ & $18(51)^{+}$ & $2(9)$ & \\
\hline Antinuclear antibodies $>1 / 160$ & $295(94)$ & $190(95)$ & 33 (94) & $22(96)$ & 0.81 \\
\hline Antitopoisomerase I antibodies & $33(11)^{\#, 9}$ & $70(35)^{+}$ & $9(26)$ & $3(13)$ & 0.006 \\
\hline Anticentromere antibodies & $203(65)^{\#, 9}$ & $45(22)^{+}$ & $9(26)^{+}$ & $12(52)$ & $<0.0001$ \\
\hline Disease duration before TO months & $92 \pm 92^{+}$ & $102 \pm 93^{+}$ & $136 \pm 104$ & $166 \pm 142$ & 0.0013 \\
\hline History of digital ulcers & $95(30)^{\pi}$ & $81(40)$ & $20(57)$ & $12(52)$ & 0.0004 \\
\hline Renal crisis & $6(2)$ & $5(2)$ & 1 (3) & $1(4)$ & 0.43 \\
\hline Calcium channel blockers & 169 (54) & 99 (49) & $16(46)$ & $13(57)$ & 0.28 \\
\hline Pulmonary hypertension specific drug therapy & $0^{\pi,+}$ & $0^{\pi,+}$ & $17(49)^{+}$ & $18(78)$ & $<0.0001$ \\
\hline Systemic corticosteroids & $41(13)^{\#, \uparrow}$ & $60(30)$ & 13 (37) & $7(30)$ & $<0.0001$ \\
\hline Immunosuppressive therapy & $34(11)^{\#, q}$ & $47(23)$ & 10 (29) & $2(9)$ & 0.0002 \\
\hline NYHA functional class & & & & & $<0.0001$ \\
\hline I & $236(75)^{\#, 9,++}$ & $83(41)^{91,+}$ & 1 (3) & $2(9)$ & \\
\hline ॥ & $65(21)^{\#, 9,+}$ & $82(41)^{91,+}$ & $22(63)$ & $9(39)$ & \\
\hline III & $12(4)^{\#, \eta_{1}+}$ & $33(16)^{1,+}$ & $12(34)$ & $10(43)$ & \\
\hline IV & $0^{\#, \uparrow,+}$ & $3(1)^{n,+}$ & 0 & $2(9)$ & \\
\hline Estimated SPAP at TO $\mathrm{mmHg}$ & $28 \pm 7^{\pi,+}$ & $30 \pm 7^{\Upsilon 1++}$ & $53 \pm 13$ & $57 \pm 19$ & $<0.0001$ \\
\hline LVEF at TO $\%$ & $64 \pm 6$ & $65 \pm 8$ & $64 \pm 6$ & $61 \pm 9$ & 0.26 \\
\hline
\end{tabular}

Data are presented as $n$, mean \pm SD or $n(\%)$. BMI: body mass index; T0: date of inclusion, i.e. date of measurement of combined transfer factor of the lung for carbon monoxide and transfer factor of the lung for nitric oxide (TLCO-TLNO); NYHA: New York Health Association; SPAP: systolic pulmonary artery pressure; LVEF: left ventricular ejection fraction. ${ }^{\#}$ : $p<0.05$ versus Group $B ;{ }^{9}: p<0.05$ versus Group $C ;{ }^{+}: p<0.05$ versus Group D.

(Group A) (table 2). In SSc patients with pulmonary hypertension (Groups C and D), VC was significantly lower than in patients without pulmonary hypertension (Groups A and B) (table 2). SSc patients with ILD (either with or without pulmonary hypertension; Groups B and C) had lower DMCO than patients without ILD (Groups A and D). It has to be noted that a total of 13 patients had a negative value of DMCO (these negative values were not taken into account in the current analysis). In addition, some patients had very high DMCO (a total of 11 patients had DMCO 200-300\% predicted and nine patients had DMCO $>300 \%$ predicted). These very high values were kept in the current analysis.

Regarding TLNO/TLCO and $D \mathrm{MCO} / V_{\mathrm{C}}$ ratios, the only significant difference found was for $D \mathrm{MCO} / V \mathrm{C}$ between SSc patients without pulmonary hypertension (Groups A and B) and SSc patients with pulmonary hypertension (Group D).

There was a strong correlation between TLCO and VC (supplementary figure S1). We also found a correlation between TLNO and TLCO, and, although weaker, between TLNO and DMCO. There was a strong nonlinear relationship between the TLNO/TLCO and the DMCO/VC ratios. However, we were unable to find any significant correlation between $\mathrm{DMCO}$ and $\mathrm{VC}$.

\section{Ability of pulmonary function tests to detect pulmonary hypertension}

Analysis of AUC values showed that TLCO (\% pred) had the highest value to detect pulmonary hypertension in our group of 572 SSc patients (figure 1 and table 3). TLNO had a slightly, but nonsignificantly lower AUC value. By contrast, $V C$ and DMCO had a significantly lower diagnostic value than both TLCO and TLNO (figure 1 and table 3). The TLNO/TLCO and the DMCO/VC ratios had the worst diagnostic values for pulmonary hypertension (figure 1 and table 3 ). 
TABLE 2 Pulmonary function tests of the studied population at inclusion, divided into four groups: systemic sclerosis (SSc) patients without any pulmonary involvement, i.e. neither interstitial lung disease (ILD) nor pulmonary hypertension (Group A), SSc patients with ILD but without pulmonary hypertension (Group B), SSc patients with both ILD and pulmonary hypertension (Group C), and SSc patients without ILD but with pulmonary hypertension (Group D)

\begin{tabular}{|c|c|c|c|c|c|}
\hline & Group A & Group B & Group C & Group D & p-value \\
\hline Subjects & 313 & 201 & 35 & 23 & \\
\hline \multicolumn{6}{|l|}{ FEV 1} \\
\hline L & $2.49 \pm 0.66^{\#, 9,++}$ & $2.28 \pm 0.63^{+}$ & $2.00 \pm 0.64$ & $1.87 \pm 0.63$ & $<0.0001$ \\
\hline$\%$ pred & $102 \pm 20^{\#, \emptyset,+}$ & $91 \pm 20$ & $83 \pm 20$ & $86 \pm 22$ & $<0.0001$ \\
\hline \multicolumn{6}{|l|}{ FVC } \\
\hline L & $3.21 \pm 0.79^{\#, 9,+}$ & $2.88 \pm 0.85$ & $2.64 \pm 0.87$ & $2.56 \pm 0.89$ & $<0.0001$ \\
\hline$\%$ pred & $111 \pm 20^{\#, \eta,+}$ & $95 \pm 22$ & $90 \pm 22$ & $97 \pm 23$ & $<0.0001$ \\
\hline FEV $/$ FVC \% lactual) & $78 \pm 8^{\#,+}$ & $80 \pm 8$ & $77 \pm 12$ & $73 \pm 7$ & $<0.0001$ \\
\hline \multicolumn{6}{|l|}{ SVC } \\
\hline L & $3.32 \pm 0.80^{\#, 9,++}$ & $2.93 \pm 0.87$ & $2.65 \pm 0.77$ & $2.52 \pm 0.68$ & $<0.0001$ \\
\hline$\%$ pred & $114 \pm 20^{\#, \eta,+}$ & $96 \pm 23$ & $91 \pm 21$ & $99 \pm 22$ & $<0.0001$ \\
\hline \multicolumn{6}{|l|}{ TLC } \\
\hline L & $5.20 \pm 0.95^{\#, 9}$ & $4.76 \pm 1.31$ & $4.63 \pm 1.13$ & $4.87 \pm 1.01$ & $<0.0001$ \\
\hline$\%$ pred & $105 \pm 15^{\#, 9}$ & $89 \pm 19$ & $86 \pm 17$ & $97 \pm 18$ & $<0.0001$ \\
\hline \multicolumn{6}{|l|}{ FRC } \\
\hline L & $3.02 \pm 0.63^{\#}$ & $2.79 \pm 0.86$ & $2.87 \pm 0.83$ & $2.83 \pm 0.73$ & 0.023 \\
\hline$\%$ pred & $109 \pm 21^{\#, 9}$ & $96 \pm 24$ & $94 \pm 22$ & $101 \pm 22$ & $<0.0001$ \\
\hline \multicolumn{6}{|l|}{ RV } \\
\hline L & $1.97 \pm 0.52^{\#}$ & $1.80 \pm 0.64^{+}$ & $1.84 \pm 0.52$ & $2.19 \pm 0.80$ & 0.001 \\
\hline$\%$ pred & $106 \pm 25^{\#, 9}$ & $91 \pm 28$ & $90 \pm 24$ & $107 \pm 38$ & $<0.0001$ \\
\hline \multicolumn{6}{|l|}{ TLCO } \\
\hline $\mathrm{mL} \cdot \mathrm{mmHg} \mathrm{g}^{-1} \cdot \mathrm{min}^{-1}$ & $18.4 \pm 5.6^{\#, 9,+}$ & $14.6 \pm 6.0^{1,+}$ & $9.6 \pm 3.3$ & $9.7 \pm 4.3$ & $<0.0001$ \\
\hline$\%$ pred & $81 \pm 22^{\#, 9,+}$ & $63 \pm 22^{\uparrow,+}$ & $44 \pm 19$ & $49 \pm 21$ & $<0.0001$ \\
\hline \multicolumn{6}{|l|}{ TLNO } \\
\hline $\mathrm{mL} \cdot \mathrm{mmHg} \mathrm{g}^{-1} \cdot \mathrm{min}^{-1}$ & $91.0 \pm 27.7^{\#, \eta_{1,+}}$ & $71.9 \pm 26.5^{1,+}$ & $51.2 \pm 18.0$ & $48.9 \pm 17.0$ & $<0.0001$ \\
\hline$\%$ pred & $67 \pm 22^{\#, 9,++}$ & $56 \pm 23^{1,+}$ & $43 \pm 17$ & $40 \pm 14$ & $<0.0001$ \\
\hline TLNO/TLCO & $5.06 \pm 1.20$ & $5.16 \pm 1.28$ & $5.46 \pm 1.42$ & $5.78 \pm 2.61$ & 0.036 \\
\hline $\mathrm{Hb} g \cdot 100 \mathrm{~mL}^{-1}$ & $13.4 \pm 1.4^{+}$ & $13.1 \pm 1.4$ & $13.0 \pm 2.0$ & $12.5 \pm 1.7$ & 0.014 \\
\hline \multicolumn{6}{|l|}{ Vc } \\
\hline $\mathrm{mL}$ & $45 \pm 18^{\#, 9,+}$ & $37 \pm 18^{\text {ๆ }}$ & $25 \pm 11$ & $27 \pm 15$ & $<0.0001$ \\
\hline$\%$ pred & $72 \pm 25^{\#, \eta,+}$ & $58 \pm 26^{7}$ & $43 \pm 23$ & $47 \pm 24$ & $<0.0001$ \\
\hline \multicolumn{6}{|l|}{ DMco } \\
\hline $\mathrm{mL} \cdot \mathrm{mmHg} \mathrm{g}^{-1} \cdot \mathrm{min}^{-1}$ & $104 \pm 105^{\#}$ & $74 \pm 38$ & $85 \pm 97$ & $105 \pm 244$ & 0.008 \\
\hline$\%$ pred & $106 \pm 101^{\#}$ & $73 \pm 38$ & $73 \pm 60$ & $138 \pm 345$ & 0.0008 \\
\hline DMco/Vc & $2.77 \pm 5.19^{+}$ & $2.38 \pm 1.87^{+}$ & $4.90 \pm 8.29$ & $7.66 \pm 24.5$ & 0.002 \\
\hline
\end{tabular}

Data are presented as $\mathrm{n}$ or mean \pm SD. $F E V 1$ : forced expiratory volume in $1 \mathrm{~s}$; FVC: forced vital capacity; SVC: slow vital capacity; TLC: total lung capacity; FRC: functional residual capacity; RV: residual volume; TLCO: transfer factor of the lung for carbon monoxide; TLNO: transfer factor of the lung for nitric oxide; $\mathrm{Hb}$ : haemoglobin concentration; $V \mathrm{C}$ : alveolar capillary blood volume; DMCO: membrane conductance (diffusing capacity) for carbon monoxide. ${ }^{\#}$ : $p<0.05$ versus Group $B_{;}{ }^{\uparrow}: p<0.05$ versus Group $C_{;}{ }^{+}: p<0.05$ versus Group D.

The sensitivities and specificities of all parameters for the detection of pulmonary hypertension are given in table 3, as well as the positive and negative predictive values for different thresholds. For the optimal thresholds determined with the Youden methods, the negative predictive values of TLCO (\% pred) and TLNO (\% pred) were very high, indicating that a TLCO and/or a TLNO above these thresholds virtually excludes pulmonary hypertension. Nevertheless, the low positive predictive values indicate that "abnormal" TLCO or TLNO were often present in the absence of pulmonary hypertension.

\section{Follow-up}

The median follow-up time for the entire SSc cohort $(n=572)$ was 24 months. The overall survival of the cohort is shown in figure 2. Patients with pulmonary hypertension (Groups C and D) had a significantly poorer survival than those without pulmonary hypertension (Groups A and B) (figure 2). Of note, also in SSc patients without pulmonary hypertension, the presence of ILD did not affect survival (figure 2). 


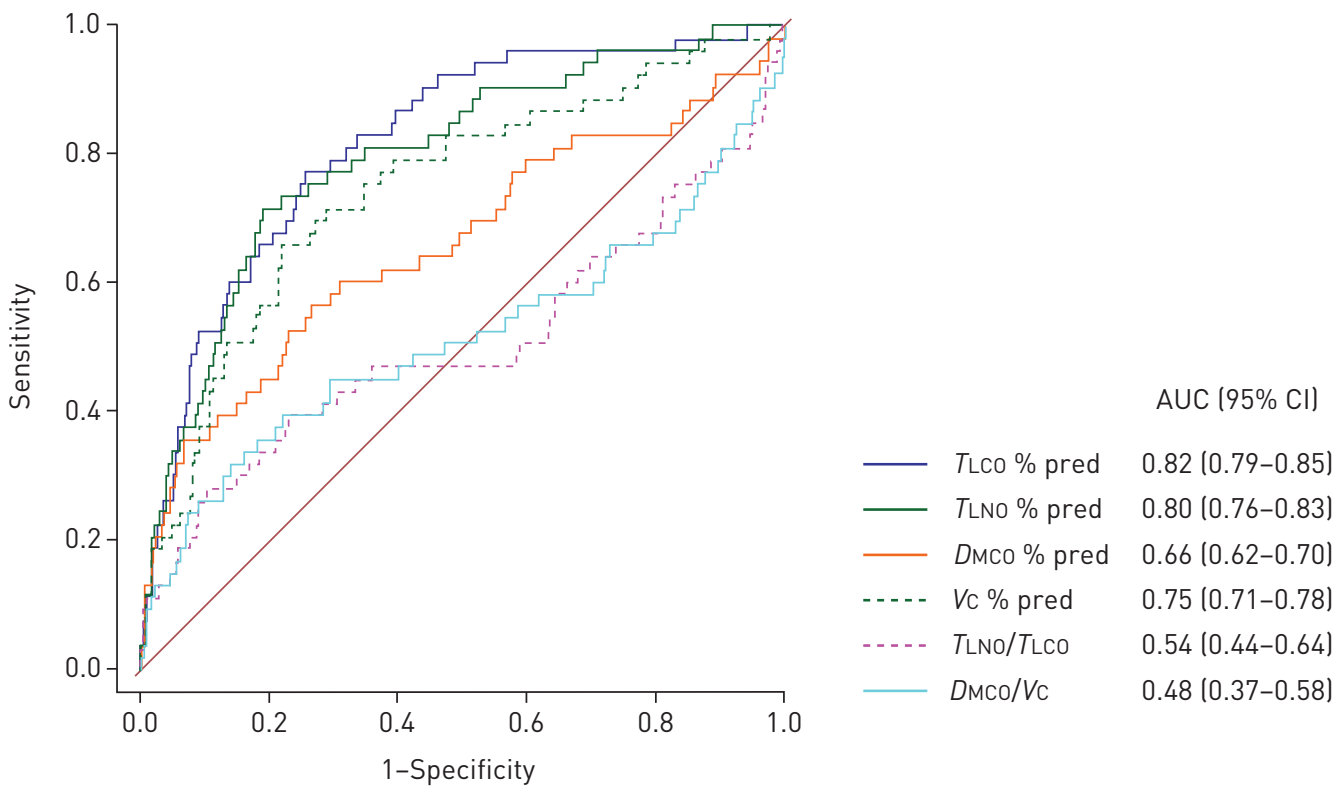

FIGURE 1 Receiver operating characteristic (ROC) curves of transfer factor of the lung for carbon monoxide (TLCO, \% pred), transfer factor of the lung for nitric oxide (TLNO), membrane conductance (diffusing capacity) for carbon monoxide (DMCO), alveolar capillary blood volume (VC), TLNO/TLCO ratio and DMCO/VC ratio for discrimination of pulmonary hypertension $(n=58)$ in comparison with the absence of pulmonary hypertension $(n=514)$ in patients with systemic sclerosis. Area under the ROC curve (AUC) values are also given.

Univariate and multivariate analyses of factors associated with survival are presented in table 4 . In univariate analysis, TLCO, TLNO, VC and DMCO were all associated with survival. In multivariate analysis, only TLCO, forced vital capacity, age at onset and pulmonary hypertension were associated with survival, with TLCO having the highest hazard ratio.

\section{Discussion}

The relevance of TLNO measurement and TLCO partitioning into DMCO and VC for the identification of pulmonary hypertension in SSc is debated, with sparse and conflicting results based on small, single-centre

\begin{tabular}{|c|c|c|c|c|c|c|}
\hline & $\operatorname{AUC}(95 \% \mathrm{CI})$ & Cut-off & Sensitivity \% & Specificity \% & PPV \% & NPV \% \\
\hline \multirow[t]{3}{*}{ TLco \% pred } & $0.82(0.79-0.85)$ & $60 \%{ }^{\#}$ & 77 & 74 & 24 & 97 \\
\hline & & LLN2.5\% & 72 & 66 & 19 & 96 \\
\hline & & LLN5\% & 78 & 58 & 17 & 96 \\
\hline \multirow[t]{3}{*}{ TLNO \% pred } & $0.80(0.76-0.83)$ & $45 \%{ }^{\#}$ & 72 & 81 & 28 & 96 \\
\hline & & LLN2.5\% & 90 & 30 & 12 & 96 \\
\hline & & LLN5\% & 93 & 24 & 12 & 97 \\
\hline \multirow[t]{3}{*}{ Vc \% pred } & $0.75(0.71-0.78)$ & $50 \%{ }^{\#}$ & 66 & 77 & 23 & 96 \\
\hline & & LLN2.5\% & 74 & 59 & 17 & 95 \\
\hline & & LLN5\% & 79 & 46 & 14 & 95 \\
\hline \multirow[t]{3}{*}{ Dмco \% pred } & $0.66(0.62-0.70)$ & $61 \%{ }^{\#}$ & 57 & 74 & 19 & 94 \\
\hline & & LLN2.5\% & 40 & 83 & 20 & 93 \\
\hline & & LLN5\% & 45 & 77 & 17 & 93 \\
\hline TLNO/TLCO & $0.54(0.44-0.64)$ & $4^{\#}$ & 19 & 94 & 26 & 92 \\
\hline DMco/VC & $0.48(0.37-0.58)$ & $1^{\#}$ & 24 & 89 & 20 & 91 \\
\hline
\end{tabular}

AUC: area under the receiver operating characteristic (ROC) curve; NPV: negative predictive value; PPV: positive predictive value; TLCO: transfer factor of the lung for carbon monoxide; LLN: lower limit of normal; LLN2.5\%: results from $2.5 \%$ of healthy individuals will be below this cut-off; LLN5\%: results from $5 \%$ of healthy individuals will be below this cut-off; TLNO: transfer factor of the lung for nitric oxide; VC: alveolar capillary blood volume; DMCO: membrane conductance (diffusing capacity) for carbon monoxide. \#: optimal threshold of pulmonary hypertension probability assessed according to the Youden index of ROC curve analysis. 


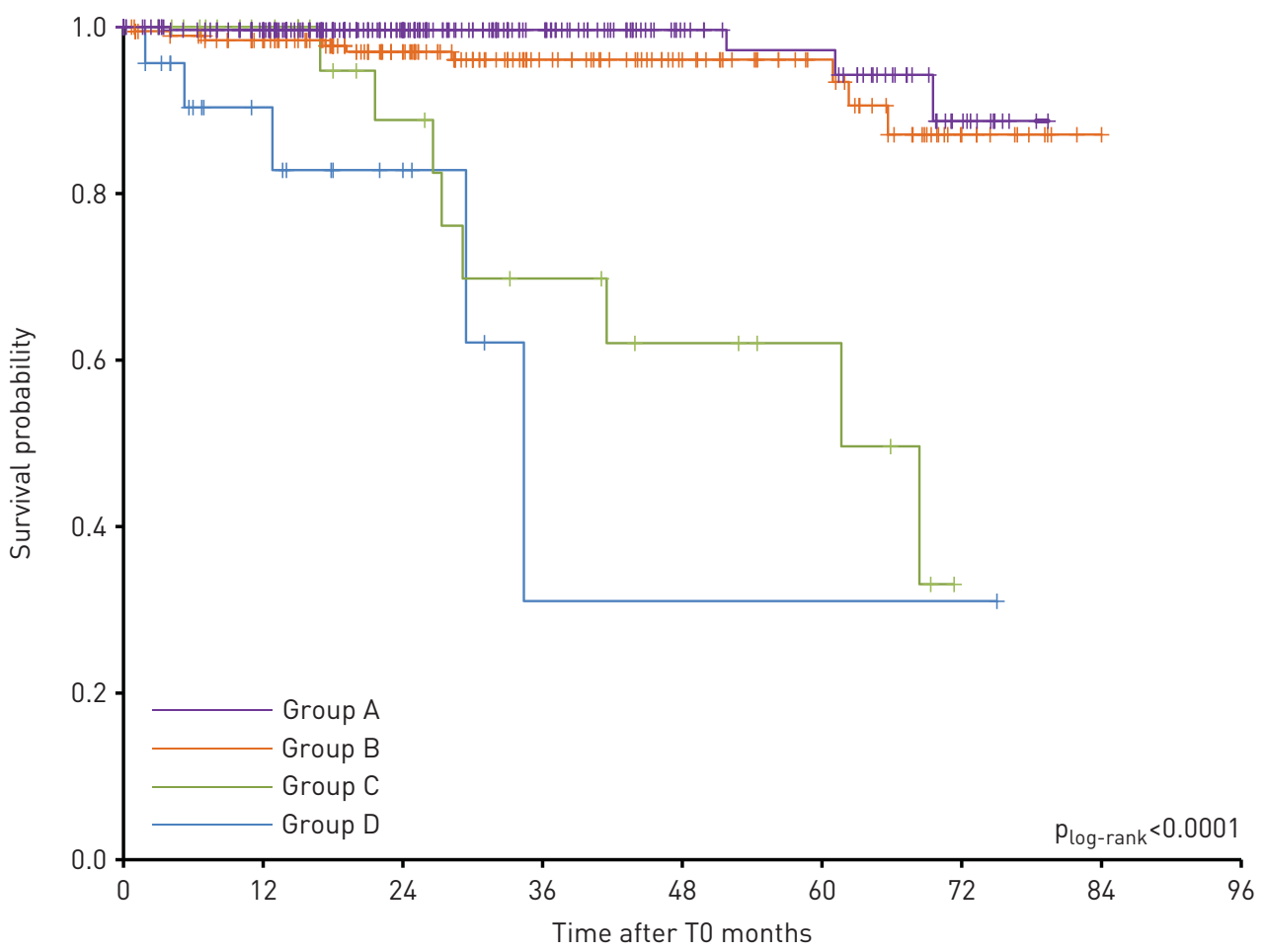

At risk $n$

$\begin{array}{ccccccccc}\text { Group A } & 313 & 249 & 145 & 80 & 46 & 33 & 11 & 0 \\ \text { Group B } & 201 & 169 & 118 & 73 & 52 & 36 & 12 & 1 \\ \text { Group C } & 35 & 24 & 15 & 10 & 7 & 5 & 0 & 0 \\ \text { Group D } & 23 & 12 & 6 & 1 & 1 & 1 & 1 & 0\end{array}$

FIGURE 2 Kaplan-Meier estimates of overall survival in patients with systemic sclerosis (SSc) but with no pulmonary involvement, i.e. neither interstitial lung disease (ILD) nor pulmonary hypertension (Group A), SSc patients with ILD but without pulmonary hypertension (Group B). SSc patients with both ILD and pulmonary hypertension (Group D), and SSc patients without ILD but with pulmonary hypertension (Group D). T0: date of inclusion, i.e. date of measurement of combined transfer factor of the lung for carbon monoxide and transfer factor of the lung for nitric oxide ( $T \mathrm{LCO}-T \mathrm{LNO})$.

series of patients [18-20]. Here, we analysed a large, multicentre series of unselected patients with well-defined SSc. Although we used recently refined equations for the calculation of VC and DMCO, we found that TLNO was equivalent to TLCO, while DMCO and VC had a lower capability than TLCO to detect pulmonary hypertension. In addition, we found that in SSc patients, TLCO was a stronger predictor of overall mortality than TLNO, VC or DMCO.

TLCO has emerged as one of the most sensitive and specific routine pulmonary function tests that have been evaluated for identifying pulmonary hypertension in patients with SSc $[10,27,28]$. This has been demonstrated in selected SSc patients: in SSc patients with TLCO $<60 \%$ predicted and disease duration $>3$ years, TLCO contributes to predicting pulmonary hypertension through the DETECT algorithm [29]. This has also been shown in unselected SSc patients, in whom TLCO contributes to predicting mean pulmonary artery pressure [30]. The analysis of our large series of unselected SSc patients confirms that TLCO is associated with the presence of pulmonary hypertension. A recent review recalled that an abnormally low TLNO, defined by a value lower than the lower limit of normal (below the 2.5th percentile (LLN2.5\%)), is more sensitive than an abnormally low TLCO to identify the presence of "cardiopulmonary diseases" [31]. We also found that a TLNO <LLN2.5\% was more sensitive than a TLCO <LLN2.5\% for the identification of pulmonary hypertension (table 3), but at the price of much lower specificity for TLNO than for TLCO.

Our study confirms that although the negative predictive value of TLCO for the presence of pulmonary hypertension is very high, the positive predictive value is poor, reflecting the fact that many patients with "low" TLCO do not have pulmonary hypertension. This is largely related to the presence of ILD, a frequent complication of SSc, which is also associated with a low TLCO [12, 32].

Although the interpretation of $V \mathrm{C}$ and $\mathrm{DMCO}$ calculated with the Roughton-Forster model remains controversial [33], these physiological parameters are supposed to discriminate between vascular and 
TABLE 4 Univariate and multivariate analyses of selected biological, clinical and functional factors associated with survival

\begin{tabular}{|c|c|c|c|c|}
\hline & \multicolumn{2}{|l|}{ Univariate } & \multicolumn{2}{|c|}{ Multivariate } \\
\hline & Hazard ratio $(95 \% \mathrm{CI})$ & p-value & Hazard ratio $(95 \% \mathrm{CI})$ & p-value \\
\hline Limited cutaneous subtype & $1.45(0.58-3.61)$ & 0.43 & & \\
\hline Age at onset $>60$ years & $2.47(1.10-5.53)$ & 0.03 & $2.79(1.23-6.35)$ & 0.01 \\
\hline Haemoglobin $<12 \mathrm{~g} \cdot \mathrm{dL}^{-1}$ & $2.24(0.97-5.16)$ & 0.06 & & \\
\hline Pulmonary hypertension & $11.40(5.27-24.66)$ & $<0.0001$ & $4.71(2.12-10.48)$ & 0.0001 \\
\hline Interstitial lung disease & $2.00(0.89-4.49)$ & 0.10 & & \\
\hline LVEF $<55 \%$ & $1.49(0.35-6.41)$ & 0.59 & & \\
\hline FVC $<$ LLN5\% & $4.49(2.06-9.79)$ & 0.0002 & $3.02(1.33-6.84)$ & 0.008 \\
\hline$\pi \mathrm{L} c 0<60 \%$ pred & 23.21 (5.48-98.39) & $<0.0001$ & $10.01(2.16-46.48)$ & 0.003 \\
\hline TLNO $<45 \%$ pred & $6.46(2.91-14.32)$ & $<0.0001$ & & \\
\hline$V c<50 \%$ pred & $7.69(2.86-20.63)$ & $<0.0001$ & & \\
\hline DMco $<61 \%$ pred & $4.93(2.07-11.73)$ & 0.0008 & & \\
\hline
\end{tabular}

LVEF: left ventricular ejection fraction; FVC: forced vital capacity; LLN: lower limit of normal; LLN5\%: results from $5 \%$ of healthy individuals will be below this cut-off; $T$ LCO: transfer factor of the lung for carbon monoxide; TLNO: transfer factor of the lung for nitric oxide; Vc: alveolar capillary blood volume; $D$ MCO: membrane conductance (diffusing capacity) for carbon monoxide.

alveolo-capillary membrane alterations. It was recently reported that $V_{C}$ is decreased in patients with pulmonary hypertension without SSc [34]. In accordance with this finding, we report that VC was lowest when pulmonary hypertension was present in SSc patients. Nevertheless, in our group of SSc patients, we also found that VC was lower in patients with ILD without pulmonary hypertension than in patients without any pulmonary involvement. Although SSc patients with ILD had preserved lung volumes and normal estimated systolic pulmonary artery pressure, our results suggest that they may have either pulmonary vascular destruction and/or pulmonary vasculopathy.

Several studies have reported a reduction in DMCO in pulmonary vascular diseases, either associated [18] or not [35] with SSc. Although thickening of the alveolo-capillary membrane due to remodelling of the pulmonary vasculature cannot be excluded, this has been previously interpreted as a loss of functional gas exchange area that accompanies the loss of $V \mathrm{C}$, a hypothesis supported by a strong correlation between $V \mathrm{C}$ and DMCO [34]. Here, we used very recently refined equations, and we did not find any significant correlation between VC and DMCO [21]. In addition, we found that DMCO was significantly lower in patients with ILD (independently of the presence of pulmonary hypertension) than in patients without. This therefore reinforces the view that DMCO provides information regarding a "thickening" of the alveolo-capillary membrane or a decrease in lung area.

An increase in TLNO/TLCO and $D \mathrm{MCO} / V \mathrm{C}$ ratios has been found in patients with pulmonary microvascular disease [36]. Although there was a tendency towards such a finding in our series of SSc patients, we found only slight differences in terms of TLNO/TLCO and $D \mathrm{MCO} / V \mathrm{C}$ ratios between patients with pulmonary hypertension and those without. Regarding the $D M C O / V C$ ratio, the absence of a significant difference between groups is likely due to the very high standard deviation, as DMCO was very high in some patients.

In agreement with previous reports, we also found here that survival of SSc patients with ILD but without pulmonary hypertension was similar to that of patients without any pulmonary involvement $[6,37]$. Nevertheless, SSc patients with pulmonary hypertension either with or without ILD had a similar poor survival. In other words, survival was linked to pulmonary hypertension rather to ILD in SSc. As prompt therapy of pulmonary hypertension in SSc has been shown to be beneficial in patients without as well as with ILD [7, 8], this reinforces the pertinence of finding a test that can identify pulmonary hypertension independently of the presence of ILD in SSc patients. Our results demonstrate that TLCO remains better than VC and DMCO calculated with the modified one-step Roughton-Forster technique for that purpose.

Strengths of this study include the real-life nature of the patient series and the multicentre assessment. Our cohort represents the largest series of patients with SSc where diffusion impairments have been assessed by combined TLCO and TLNO measurements. The group of unselected SSc patients analysed here is likely to be representative of SSc patients followed in most referral centres because in the seven participating centres, each patient with SSc has an annually scheduled follow-up that comprises echocardiography and pulmonary function tests. $41 \%$ of our patients had some degree of ILD; this percentage is similar to that reported in previous SSc cohorts [38] and ILD was more than twice as 
common among patients with diffuse cutaneous subtype than among those with limited subtype. The prevalence of pulmonary hypertension $(10.1 \%)$ found here is also close to that reported in previous series $[3,11,39,40]$.

Limitations of this study include the retrospective analysis and the observational nature of our cohort. We were therefore unable to identify in each of the seven participating centres the number of SSc patients who were not included in the current study because of missing data on echocardiography and/or right heart catheterisation and/or HRCT and/or pulmonary function tests. There are also limitations in terms of the assessments that were directed by clinical practice and did not follow a predefined study protocol. Some important complications were not ascertained (e.g. gastrointestinal tract involvement) and some others were impossible to collect in some centres (e.g. Rodnan score, which assesses skin thickness in the diffuse cutaneous subtype of SSc). We were unable to collect haemodynamic data for all patients who underwent right heart catheterisation; these data were therefore omitted and only the item "pre-capillary pulmonary hypertension according to right heart catheterisation" was collected in all patient files. Nevertheless, echocardiographic estimation of systolic pulmonary artery pressure was systematically reviewed and collected.

In conclusion, our study provides evidence that the lower TLCO in SSc patients with pulmonary hypertension, compared with SSc patients without pulmonary hypertension, is attributable to the lower VC in those with pulmonary hypertension. Nevertheless, we failed to demonstrate any clinical usefulness of the TLCO partition into DMCO and VC for the identification of pulmonary hypertension in patients with SSc. Our results are at variance with some results based on small and/or selected populations, thus highlighting the importance of cohort representativeness for the applicability of study results to a general population of SSc patients.

\section{References}

1 Dumoitier N, Lofek S, Mouthon L. Pathophysiology of systemic sclerosis: state of the art in 2014. Presse Med 2014; 43: e267-e278.

2 Steen VD, Medsger TA. Changes in causes of death in systemic sclerosis, 1972-2002. Ann Rheum Dis 2007; 66: 940-944.

3 Solomon JJ, Olson AL, Fischer A, et al. Scleroderma lung disease. Eur Respir Rev 2013; 22: 6-19.

4 Goh NS, Desai SR, Veeraraghavan S, et al. Interstitial lung disease in systemic sclerosis: a simple staging system. Am J Respir Crit Care Med 2008; 177: 1248-1254.

5 Wells AU, Cullinan P, Hansell DM, et al. Fibrosing alveolitis associated with systemic sclerosis has a better prognosis than lone cryptogenic fibrosing alveolitis. Am J Respir Crit Care Med 1994; 149: 1583-1590.

6 Bouros D, Wells AU, Nicholson AG, et al. Histopathologic subsets of fibrosing alveolitis in patients with systemic sclerosis and their relationship to outcome. Am J Respir Crit Care Med 2002; 165: 1581-1586.

7 Humbert M, Yaici A, de Groote P, et al. Screening for pulmonary arterial hypertension in patients with systemic sclerosis: clinical characteristics at diagnosis and long-term survival. Arthritis Rheum 2011; 63: 3522-3530.

8 Volkmann ER, Saggar R, Khanna D, et al. Improved transplant-free survival in patients with systemic sclerosis-associated pulmonary hypertension and interstitial lung disease. Arthritis Rheumatol 2014; 66: 1900-1908.

9 Khanna D, Gladue H, Channick R, et al. Recommendations for screening and detection of connective tissue disease-associated pulmonary arterial hypertension. Arthritis Rheum 2013; 65: 3194-3201.

10 Steen V, Medsger TA Jr. Predictors of isolated pulmonary hypertension in patients with systemic sclerosis and limited cutaneous involvement. Arthritis Rheum 2003; 48: 516-522.

11 Hachulla E, Gressin V, Guillevin L, et al. Early detection of pulmonary arterial hypertension in systemic sclerosis: a French nationwide prospective multicenter study. Arthritis Rheum 2005; 52: 3792-3800.

12 Pernot J, Puzenat E, Magy-Bertrand N, et al. Detection of interstitial lung disease in systemic sclerosis through partitioning of lung transfer for carbon monoxide. Respiration 2012; 84: 461-468.

13 Roughton FJ, Forster RE. Relative importance of diffusion and chemical reaction rates in determining rate of exchange of gases in the human lung, with special reference to true diffusing capacity of pulmonary membrane and volume of blood in the lung capillaries. J Appl Physiol 1957; 11: 290-302.

14 Degano B, Mittaine M, Guénard H, et al. Nitric oxide and carbon monoxide lung transfer in patients with advanced liver cirrhosis. J Appl Physiol 2009; 107: 139-143.

15 Guénard H, Varene N, Vaida P. Determination of lung capillary blood volume and membrane diffusing capacity in man by the measurements of NO and CO transfer. Respir Physiol 1987; 70: 113-120.

16 Zavorsky GS, Hsia CC, Hughes JM, et al. Standardisation and application of the single-breath determination of nitric oxide uptake in the lung. Eur Respir J 2017; 49: 1600962.

17 Zavorsky GS. Nitric oxide uptake in the lung: it is about time that clinicians use this test routinely. Respir Physiol Neurobiol 2017; 241: 1-2.

18 Overbeek MJ, Groepenhoff H, Voskuyl AE, et al. Membrane diffusion- and capillary blood volume measurements are not useful as screening tools for pulmonary arterial hypertension in systemic sclerosis: a case control study. Respir Res 2008; 9: 68.

19 Guarnieri G, Zanatta E, Mason P, et al. Determinants of impairment in lung diffusing capacity in patients with systemic sclerosis. Clin Exp Rheumatol 2015; 33: S80-S86.

20 Sivova N, Launay D, Wemeau-Stervinou L, et al. Relevance of partitioning DLCO to detect pulmonary hypertension in systemic sclerosis. PLoS One 2013; 8: e78001.

21 Martinot JB, Guénard H, Dinh-Xuan AT, et al. Nitrogen monoxide and carbon monoxide transfer interpretation: state of the art. Clin Physiol Funct Imaging 2017; 37: 357-365. 
22 van den Hoogen F, Khanna D, Fransen J, et al. 2013 classification criteria for systemic sclerosis: an American College of Rheumatology/European League against Rheumatism collaborative initiative. Arthritis Rheum 2013; 65: 2737-2747.

23 Miller MR, Hankinson J, Brusasco V, et al. Standardisation of spirometry. Eur Respir J 2005; 26: 319-338.

24 Wanger J, Clausen JL, Coates A, et al. Standardisation of the measurement of lung volumes. Eur Respir J 2005; 26: 511-522.

25 Quanjer PH, Tammeling GJ, Cotes JE, et al. Lung volumes and forced ventilatory flows. Report Working Party Standardization of Lung Function Tests, European Community for Steel and Coal. Official Statement of the European Respiratory Society. Eur Respir J Suppl 1993; 6: Suppl. 16, 5-40.

26 Youden WJ. Index for rating diagnostic tests. Cancer 1950; 3: 32-35.

27 Salerni R, Rodnan GP, Leon DF, et al. Pulmonary hypertension in the crest syndrome variant of progressive systemic sclerosis (scleroderma). Ann Intern Med 1977; 86: 394-399.

28 Stupi AM, Steen VD, Owens GR, et al. Pulmonary hypertension in the crest syndrome variant of systemic sclerosis. Arthritis Rheum 1986; 29: 515-524.

29 Coghlan JG, Denton CP, Grunig E, et al. Evidence-based detection of pulmonary arterial hypertension in systemic sclerosis: the DETECT study. Ann Rheum Dis 2014; 73: 1340-1349.

30 Schreiber BE, Valerio CJ, Keir GJ, et al. Improving the detection of pulmonary hypertension in systemic sclerosis using pulmonary function tests. Arthritis Rheum 2011; 63: 3531-3539.

31 Zavorsky GS, van der Lee I. Can the measurement of pulmonary diffusing capacity for nitric oxide replace the measurement of pulmonary diffusing capacity for carbon monoxide? Respir Physiol Neurobiol 2017; 241: 9-16.

32 Wells AU, Hansell DM, Rubens MB, et al. Fibrosing alveolitis in systemic sclerosis: indices of lung function in relation to extent of disease on computed tomography. Arthritis Rheum 1997; 40: 1229-1236.

33 Kang MY, Grebenkov D, Guénard H, et al. The Roughton-Forster equation for $\mathrm{DL}_{\mathrm{CO}}$ and $\mathrm{DL}_{\mathrm{NO}}$ re-examined. Respir Physiol Neurobiol 2017; 241: 62-71.

34 Godinas L, Amar D, Montani D, et al. Lung capillary blood volume and membrane diffusion in precapillary pulmonary hypertension. J Heart Lung Transplant 2016; 35: 647-656.

35 Farha S, Laskowski D, George D, et al. Loss of alveolar membrane diffusing capacity and pulmonary capillary blood volume in pulmonary arterial hypertension. Respir Res 2013; 14 : 6.

36 Hughes JMB, Dinh-Xuan AT. The $\mathrm{DL}_{\mathrm{NO}} / \mathrm{DL}_{\mathrm{CO}}$ ratio: physiological significance and clinical implications. Respir Physiol Neurobiol 2017; 241: 17-22.

37 Cappelli S, Bellando Randone S, Camiciottoli G, et al. Interstitial lung disease in systemic sclerosis: where do we stand? Eur Respir Rev 2015; 24: 411-419.

38 Nihtyanova SI, Schreiber BE, Ong VH, et al. Prediction of pulmonary complications and long-term survival in systemic sclerosis. Arthritis Rheum 2014; 66: 1625-1635.

39 Avouac J, Airo P, Meune C, et al. Prevalence of pulmonary hypertension in systemic sclerosis in European Caucasians and metaanalysis of 5 studies. J Rheumatol 2010; 37: 2290-2298.

40 Morrisroe K, Huq M, Stevens W, et al. Risk factors for development of pulmonary arterial hypertension in Australian systemic sclerosis patients: results from a large multicenter cohort study. BMC Pulm Med 2016; 16 : 134 